\title{
Delayed use of eribulin in a heavily pretreated liposarcoma patient, previously misdiagnosed as leiomyosarcoma
}

\author{
Federica Martorana*,1,2 (D), Paolo Vigneri ${ }^{1,2,3}$, Livia Manzella ${ }^{2,3}$, Elena Tirrò ${ }^{2,3}$ \& Héctor J. \\ Soto Parra ${ }^{1}$ \\ ${ }^{1}$ Division of Medical Oncology, A.O.U. Policlinico-Vittorio Emanuele, Catania, Italy \\ ${ }^{2}$ Center of Experimental Oncology \& Hematology, A.O.U. Policlinico-Vittorio Emanuele, Catania, Italy \\ ${ }^{3}$ Department of Clinical \& Experimental Medicine, University of Catania, Catania, Italy \\ *Author for correspondence: Tel.: +39 095378 1516; fede.marto.fm@gmail.com
}

Due to its low incidence, liposarcoma displays a limited number of therapeutic options. However, eribulin recently received approval for the treatment of advanced liposarcoma patients, progressing to at least two chemotherapy lines. We report herein the case of a man initially diagnosed with a leyomiosarcoma, subsequently reclassified as a dedifferentiated liposarcoma, who received eribulin after he failed several therapy lines. Eribulin provided our patient an 8-month disease control and a substantial clinical benefit with no relevant adverse effects, showing a good efficacy and safety profile despite its delayed employ. Additionally, this case strengthens the pivotal importance of molecular profiling in the management of soft tissue sarcomas.

First draft submitted: 24 September 2019; Accepted for publication: 11 December 2019; Published online: 9 January 2020

Keywords: eribulin • liposarcoma • molecular profiling

Eribulin mesylate (EM), a synthetic analog of halichondrin B, a drug isolated from the sea sponge Halichondria okadai, is a microtubule inhibitor approved for the treatment of metastatic breast cancer and liposarcoma. EM binds microtubule ends, leading to the assemblance of aberrant mitotic spindles, which are unable to pass through the metaphase/anaphase checkpoint. This eventually determines cell cycle arrest and apoptosis [1]. Additionally, eribulin seems also to affect angiogenesis and micro-environment, by causing vascular remodeling and increasing neoplastic perfusion [2]. In a Phase II study conducted on 128 patients with progressive or high-grade soft-tissue sarcoma of unselected histology, eribulin showed encouraging activity in adipocytic sarcoma and leiomyosarcoma [3]. In a subsequent randomized, open label, multicentric Phase III trial (E7389-G000-309), which compared EM with dacarbazine in 452 patients with advanced liposarcoma or leiomyosarcoma, who had already received at least two lines of therapy, eribulin significantly improved survival (overall survival [OS]; 13.5 vs 11.3 months, hazard ratio [HR]: 0.75 ; 95\% CI: $10.9-15.6)$ but not progression-free survival (PFS; 2.6 vs 2.6 months, hazard ratio: $0.86 ; 95 \%$ CI: 1.9-2.8) in the overall population. However, a prespecified exploratory subgroup analysis showed a substantial benefit both in OS and PFS for only for patients with the liposarcoma histotype [4,5]. Hence, in 2016 both the US FDA and the European Medicine Agency approved EM for the treatment of patients with advanced liposarcoma that had previously received an anthracycline.

Here we report the case of a patient with a dedifferentiated liposarcoma, initially misdiagnosed as a high-grade leiomyosarcoma, who progressed after several lines of therapy before experiencing disease stabilization with EM.

\section{Case presentation}

In August 2012, a white Caucasian 55-year-old male subject with no relevant co-morbidities came to our attention complaining of persistent abdominal pain and intermittent constipation. A contrast enhanced CT scan and an MRI (Figure 1) revealed the presence of a $10 \times 4 \mathrm{~cm}$ retroperitoneal mass that after the surgical excision was characterized as a high-grade leiomyosarcoma with immunohistochemical staining positive for CD34 and desmin, and with a

Future $\because$ Medicine 


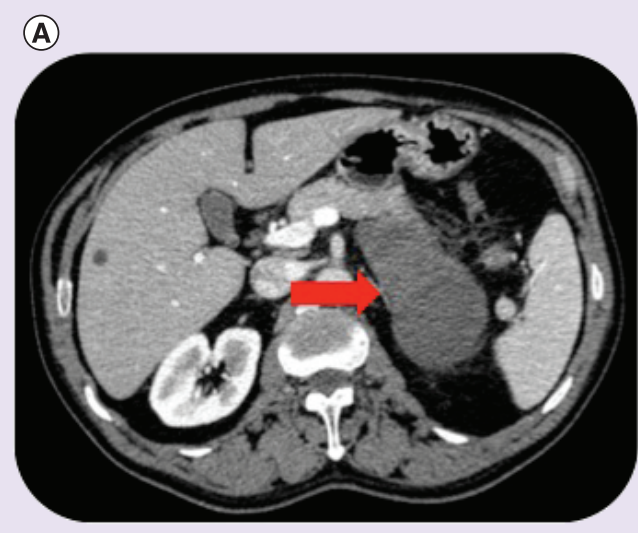

(B)

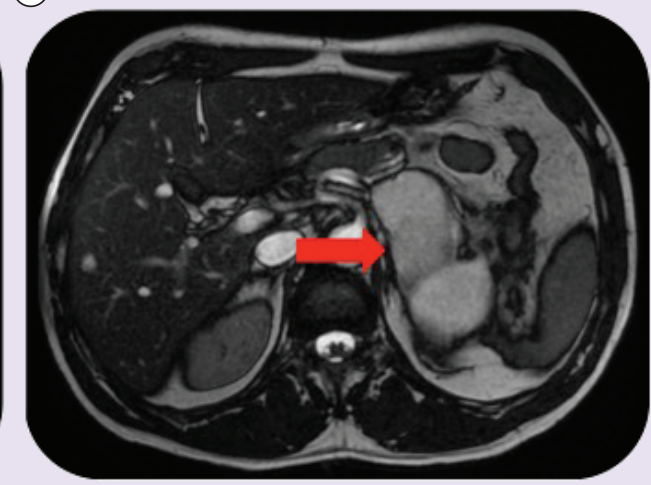

Figure 1. Imaging at diagnosis. CT scan (A) and MRI (B) showed a mass in the anterior para-renal space, behind the pancreatic tail, taking contact with the spleen, the splenic vessels, the descending colon and the bowel.

$45 \%$ of Ki-67-positive cells. Given the high risk of recurrence, the patient received four courses of adjuvant epirubicin $90 \mathrm{mg} / \mathrm{m}^{2}$ on day 1 and dacarbazine $300 \mathrm{mg} / \mathrm{m}^{2}$ on days $1-3$ of each 21 days cycle, from December 2012 to February 2013, and then began regular follow-up assessments. He remained disease-free until March 2014, when a positron-emission tomography scan showed three new asymptomatic lesions in the left hypochondrium. At this stage, the patient underwent a second surgical procedure that included a distal pancreasectomy, a partial gastrectomy and a segmental left colectomy, thus achieving an R0 excision. The pathologist's assessment confirmed the previous diagnosis of poorly differentiated leiomyosarcoma. Follow-up controls remained unremarkable until a CT scan performed in June 2015 revealed a bulky sub-diaphragmatic mass measuring $10 \times 7 \mathrm{~cm}$, along with two satellite lesions of $4.7 \times 2.5 \mathrm{~cm}$ and $4 \times 2 \mathrm{~cm}$. As the referral surgeon did not deem the relapse amenable to further excision, in July 2015 the patient started a first-line systemic treatment with a combination of gemcitabine $900 \mathrm{mg} / \mathrm{m}^{2}$ on days 1 and 8 and docetaxel $75 \mathrm{mg} / \mathrm{m}^{2}$ on day 8 every 3 weeks. This regimen was not well tolerated as the patient experienced persistent grade 2 nausea and fatigue, as well as recurrent grade 2 thrombocytopenia and an episode of febrile neutropenia. However, a CT scan performed after 3 months of therapy showed disease of progression according to RECIST 1.1 criteria, with a dimensional increase of the solid component in all the lesions and the evidence of a new nodule of $2 \times 1 \mathrm{~cm}$ in the left flank [6]. We, therefore, opted for a second-line treatment with continuous infusion of ifosfamide $1000 \mathrm{mg} / \mathrm{m}^{2}$ daily for 14 days every 28 days, which resulted in a 10-month disease control without any relevant toxicity. In November 2016, an additional scan revealed a $25 \%$ increase in the size of the main lesion and the patient, still asymptomatic, began the treatment with trabectedin $1.5 \mathrm{mg} / \mathrm{m}^{2}$ over a $24 \mathrm{~h}$ continuous infusion every 3 weeks, from which he gained no benefit, followed by pazopanib $800 \mathrm{mg} /$ daily, until June 2017. Unfortunately, at this time, he presented to the Emergency Room because of acute abdominal pain following 1 week of constipation. Abdomen $\mathrm{x}$-rays showed multiple air-fluid levels with expansion of the intestinal loops, consistent with bowel obstruction. Thus, he underwent palliative debulking surgery with small bowel resection. Even though the surgeon successfully removed a large part of the underlying neoplastic mass, the resection was not radical, with gross residual disease in the left hypochondrium. Unexpectedly, the pathologist's evaluation indicated the presence of a dedifferentiated liposarcoma, 85\% of which was well differentiated (i.e., lipoma-like and sclerosant sarcoma) and 15\% dedifferentiated (i.e., epithelioid/rhabdoid and spindle cell sarcoma with focal myogenic differentiation and pleomorphic liposarcoma). Immunohistochemical staining was positive for $\alpha$-actin (1A4) and INI1, whereas it was negative for desmin and myogenin. In order to confirm this new histological finding, we requested a revision of the original 2012 histological specimen, which was indeed reclassified as a poorly differentiated liposarcoma with myogenic differentiation. Additionally, we decided to molecularly characterize the disease employing the formalin fixed-paraffin-embedded sample from the most recent surgery and we found $C D K 4, M D M 2$ and FRS2 amplification along with $H M G A 2$ translocation. These findings further corroborated the diagnosis of dedifferentiated liposarcoma. After the palliative surgery, the patient asked for a therapeutic pause, and we decided to strictly monitor the residual disease and postpone a new active treatment to symptomatic progression. Although two consecutive CT scans performed in August and October 2017 showed 

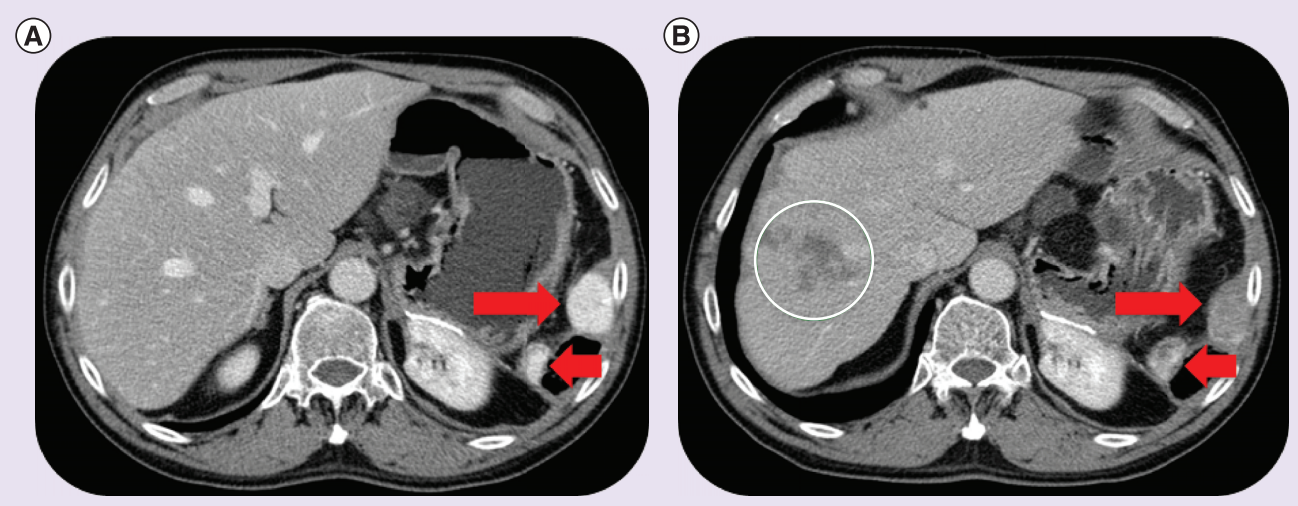

Figure 2. CT scan at baseline (A) and after 4 cycles of eribulin (B). While the two lesions in the left flank did not display substantial shrinkage, their modified contrast enhancement was indicative of intra-tumoral necrosis. The hepatic region circled in green in $\mathrm{B}$ is a pre-existing angioma.

a small increase in tumor size, the patient remained off therapy and asymptomatic until January 2018, when he started complaining of mild abdominal pain, that was responsive to tramadol. At this time, after an additional radiological evaluation confirmed a significant disease progression, we began a fifth-line treatment with eribulin $1.23 \mathrm{mg} / \mathrm{m}^{2}$ on days 1 and 8 of a 21-day cycle. At the end of the second cycle, the patient reported subjective clinical improvement and pain relief that led to tramadol discontinuation. The first radiological evaluation, performed with a CT scan after the fourth cycle, showed substantial disease stability with a remarkable modification in the main lesion's contrast enhancement due to diffuse intratumoral necrosis (Figure 2). The disease remained stable after completing eight cycles of treatment, as confirmed by a CT scan performed in July 2018. Treatment with EM was well tolerated, except for grade 1 fatigue, grade 2 alopecia, grade 2 nausea (successfully treated with alizapride and low-dose dexamethasone) and a single episode of grade 3 neutropenia that resolved without the use of G-CSF. However, in August 2018 the patient displayed a progressive worsening of his general health condition, reporting asthenia, anorexia, abdominal discomfort and bloating with occasional episodes of breakthrough pain. Therefore, we decided to perform an early radiological evaluation, which revealed a massive progression of the abdominal disease and the appearance of liver metastases. Hence, we stopped the EM treatment and activated palliative care. The patient eventually died of pulmonary thromboembolism on 3 January 2019.

\section{Molecular analysis}

In order to further confirm the second diagnosis of dedifferentiated liposarcoma, we decided to perform a comprehensive molecular analysis of the tissue specimen using the hybrid capture-based next generation sequencing (FoundationOne ${ }^{\circledR}$ Heme). This assay performs DNA sequencing to analyze 406 genes and selected introns of 31 genes involved in rearrangements, along with RNA sequencing of 265 genes. The average median exon coverage for the sample was 834X [7]. CCND3, CDK4, FRS2, MDM2 and mTOR amplification, HMGA2-DNM3OS gene fusion, an $S M O$ point mutation and TET2 truncation emerged in the sample from the next generation sequencing (NGS) analysis.

These results were mainly consistent with the diagnosis of dedifferentiated liposarcoma, since $C D K 4, M D M 2$ and FRS2 amplification and HMGA2 translocation involve chromosome 12q14-15. Even though the above-mentioned alterations have already been identified as distinctive features of dedifferentiated liposarcoma, this is to the best of our knowledge the first time CDK4, MDM2, FRS2 amplification and HMGA2 translocation are reported altogether in the same tumor [8-11]. However, HMGA2 translocations have never been identified in liposarcomas before. Additionally, $C C N D 3$ and $m T O R$ amplifications are novel findings in dedifferentiated liposarcoma and, although it is impossible to determine whether they represent diver events in cancer survival and progression, they may indicate a loss of cell-cycle regulation in this tumor. Similarly, SMO and TET2 alterations are not typical of liposarcoma, albeit they are frequent in other tumor types, such as skin tumors or leukemia, respectively $[12,13]$.

Taken together, molecular characterization of the tumor not only supported the correct re-definition of histopathological diagnosis, but also shed light on some unexpected genomic features. The high number of 
alterations identified may be explained as a result of clonal evolution and selective pressure of cancer therapies in a heavily pretreated patient, determining genomic heterogeneity and instability. In this context it remains controversial, if not impossible, to distinguish driver from passenger alterations, in order to select those that can be more effectively targeted.

\section{Discussion \& conclusion}

As soft tissue sarcomas are a heterogeneous group of extremely infrequent disorders with an unfavorable prognosis and limited therapeutic options, the search for new effective drugs to treat these aggressive diseases represents an unmet medical need. Furthermore, in order to pursue a personalized treatment approach, it is essential to better classify these tumors according to their intrinsic molecular characteristics, regardless of their morphology [14]. In the above-mentioned scenario, EM plays a pivotal role in the therapeutic strategy for high-grade liposarcoma patients. Indeed, in the E7389-G000-309 trial described above EM treatment displayed a significant efficacy and an adequate safety profile in heavily pretreated patients [4].

In our case, morphology- and immunohistochemistry-based pathological assessments were initially misleading, causing improper therapeutic choices (i.e., pazopanib) and a delay in EM administration. It is evident that the intervention of a dedicated pathologist, along with the use of molecular analyses, could avoid unnecessary and potentially harmful therapies and should, therefore, be considered in every case of newly diagnosed soft tissue sarcomas.

However, while molecular characterization of soft tissue sarcoma has a pivotal role for a precise diagnostic definition of this heterogeneous group of diseases, its role in the identification of potential therapeutic targets remains controversial. In fact, results from several studies seem to be disappointing in terms of number of actionable alteration identified, number of patients eventually treated with targeted agents and response rate to these therapies [15]. Nonetheless, the prospective randomized MULTISARC trial (NCT03784014) is currently ongoing to define whether molecular analysis deserves a role in treatment definition for patients with sarcoma [16].

Hence, it is impossible to determine whether exploiting one or more of the molecular alterations that we found in our patient as a therapeutic target would have led to a clinical benefit or an extended survival.

In the present report, we describe a patient that achieved clinical benefit in terms of both an 8-month PFS and a quality-of-life improvement after receiving EM as a fifth-line treatment option. This probably explains why, although the patient's PFS was considerably longer than that reported in the E7389-G000-309 trial (2.9 months in the liposarcoma subgroup), his OS was shorter (11.2 vs 15.6 months, as obtained in the trial). Hence, we can hypothesize that anticipating EM administration in an earlier line would have had a greater impact on a patient's survival. Moreover, the toxicities experienced by our patient were in line with those reported in literature, confirming a favorable safety profile even in a pretreated patient.

In fact, in E7389-G000-309, 32, 39 and 24\% of patients receiving EM experienced grade 1-2, 3 and 4 adverse event, respectively. The most common toxicities of any grade in the EM arm were fatigue ( $41 \%$ grade $1-2,3 \%$ grade 3 ), nausea (39\% grade $1-2,1 \%$ grade 3$)$ and alopecia (39\% grade $1-2)$, whereas most frequent grade 3 $(20 \%)$ and grade $4(15 \%)$ adverse event was neutropenia [4].

In conclusion, our experience confirms that eribulin represents a viable, histology driven, therapeutic option for advanced liposarcoma. Even if our clinical case demonstrates a reasonable activity and good tolerability of the drug in a heavily pretreated patient, it is advisable to anticipate EM administration in order to maximize its potential efficacy. Moreover, several trials are currently evaluating safety and efficacy of EM in combination with different agents in various sarcoma subtypes, including liposarcoma. While a Korean (NCT03810976) Phase II trial is testing EM with the conventional chemotherapy agent gemcitabine, two additional studies are currently exploring more innovative combinations with the multikinase inhibitor Lenvatinib (Phase I/II, NCT03526679) and with the anti-PD-1 immune-checkpoint inhibitor Pembrolizumab (Phase II, NCT03899805). Hence, in the future, EM activity could be enhanced by its employ in combination regimens.

Author contributions

All authors were involved in the writing and revision of this manuscript and have provided final approval to submit.

Disclaimer

The supporting company was not offered the opportunity to revise the manuscript and had no role in the decision to submit this report to the Journal. 


\section{Financial \& competing interests disclosure}

The authors have no relevant affiliations or financial involvements with any organization or entity with a financial interest in or a financial conflict with the subject matter or materials discussed in the manuscript. This includes employment, consultancies, honoraria, stock ownership or options, expert testimony, grants or patents received or pending, or royalties.

Medical writing support was provided by Amalia Forte, PhD, on behalf of Content Ed Net, and funded by Eisai. Eisai supported the editorial assistance.

\section{Ethical conduct of research}

The authors state that they have followed the principles outlined in the Declaration of Helsinki for all human or animal experimental investigations. In addition, for investigations involving human subjects, informed consent has been obtained from the patients to collect data for scientific publications concerning their cases.

\section{References}

Papers of special note have been highlighted as: $\bullet$ of interest; $\bullet \bullet$ of considerable interest

1. Okouneva T, Azarenko O, Wilson L, Littlefield BA, Jordan MA. Inhibition of centromere dynamics by eribulin (E7389) during mitotic metaphase. Mol. Cancer Ther. 7(7), 2003-2011 (2008).

2. Dybdal-Hargreaves NF, Risinger AL, Mooberry SL. Eribulin mesylate: mechanism of action of a unique microtubule-targeting agent. Clin. Cancer Res. 21(11), 2445-2452 (2015).

3. Kawai A, Araki N, Naito Y et al. Phase II study of eribulin in patients with previously treated advanced or metastatic soft tissue sarcoma. Jpn J. Clin. Oncol. 47(2), 137-144 (2016).

4. Schöffski P, Chawla S, Maki RG et al. Eribulin versus dacarbazine in previously treated patients with advanced liposarcoma or leiomyosarcoma: a randomised, open-label, multicentre, Phase III trial. Lancet 387(10028), 1629-1637 (2016).

5. Demetri GD, Schöffski P, Grignani G et al. Activity of eribulin in patients with advanced liposarcoma demonstrated in a subgroup analysis from a randomized Phase III study of eribulin versus dacarbazine. J. Clin. Oncol. 35(30), 3433-3439 (2017).

6. Eisenhauer EA, Therasse P, Bogaerts J et al. New response evaluation criteria in solid tumours: revised RECIST guideline (version 1.1). Eur. J. Cancer 45(2), 228-247 (2009).

7. Frampton GM, Fichtenholtz A, Otto GA et al. Development and validation of a clinical cancer genomic profiling test based on massively parallel DNA sequencing. Nat. Biotechnol. 31(11), 1023 (2013).

8. Mandahl N, Magnusson L, Nilsson J et al. Scattered genomic amplification in dedifferentiated liposarcoma. Mol. Cytogenet. 10(1), 25 (2017).

9. Ricciotti RW, Baraff AJ, Jour G et al. High amplification levels of MDM2 and CDK4 correlate with poor outcome in patients with dedifferentiated liposarcoma: a cytogenomic microarray analysis of 47 cases. Cancer Genet. 218, 69-80 (2017).

10. Saâda-Bouzid E, Burel-Vandenbos F, Ranchère-Vince D et al. Prognostic value of HMGA2, CDK4, and JUN amplification in well-differentiated and dedifferentiated liposarcomas. Mod. Pathol. 28(11), 1404 (2015).

11. Zhang K, Chu K, Wu X et al. Amplification of FRS2 and activation of FGFR/FRS2 signaling pathway in high-grade liposarcoma. Cancer Res. 73(4), 1298-1307 (2013).

12. Kim HS, Kim YS, Lee C, Shin MS, Kim JW, Jang BG. Expression profile of sonic hedgehog signaling-related molecules in basal cell carcinoma. PLoS ONE 14(11), e0225511 (2019).

13. Chiba S. Dysregulation of TET2 in hematologic malignancies. Int. J. Hematol. 105(1), 17-22 (2017).

14. Brenca M, Maestro R. Massive parallel sequencing in sarcoma pathobiology: state of the art and perspectives. Exp. Rev. Anticancer Ther. 15(12), 1473-1488 (2015).

15. Dufresne A, Brahmi M, Karanian M, Blay JY. Using biology to guide the treatment of sarcomas and aggressive connective-tissue tumours. Nat. Rev. Clin. Oncol. 15(7), 443-458 (2018).

16. Italiano A. Is there value in molecular profiling of soft-tissue sarcoma?. Curr. Treat. Options Oncol. 19(12), 78 (2018). 